\title{
Erratum: Linear and nonlinear magnetic error measurements using action and phase jump analysis [Phys. Rev. ST Accel. Beams 12, 014002 (2009)]
}

\author{
Javier F. Cardona* \\ National University of Colombia, Ciudad Universitaria, Bogotá, Colombia \\ Stephen G. Peggs \\ Brookhaven National Laboratory, Upton, New York 11973, USA \\ (Received 21 April 2010; published 11 May 2010)
}

DOI: 10.1103/PhysRevSTAB.13.059901

PACS numbers: 41.85.-p, 29.85.Fj, 29.20.-c, 99.10.Cd

Some typographical errors have been detected in several equations in our paper by Blanco. The first error appears in Eq. (10): We missed root square symbols for terms $2 J_{0}$. The equation should read

$$
\tan \delta_{1}=\frac{\theta_{z} \sqrt{\beta_{z}\left(s_{\theta}\right)} \sin \left[\psi_{z}\left(s_{\theta}\right)\right]+\sqrt{2 J_{0}} \sin \delta_{0}}{\theta_{z} \sqrt{\beta_{z}\left(s_{\theta}\right)} \cos \left[\psi_{z}\left(s_{\theta}\right)\right]+\sqrt{2 J_{0}} \cos \delta_{0}} .
$$

The second error appears in Eqs. (13) and (14): We missed square root symbols for terms $\beta_{z i}$ and $\beta_{z i+1}$. The equation should read

$$
J_{i+1}=\frac{\left(z_{i} / \sqrt{\beta_{z_{i}}}\right)^{2}+\left(z_{i+1} / \sqrt{\beta_{z_{i+1}}}\right)^{2}}{2 \sin ^{2}\left(\psi_{z_{i+1}}-\psi_{z_{i}}\right)}-\frac{z_{i} z_{i+1} \cos \left(\psi_{z_{i+1}}-\psi_{z_{i}}\right)}{\sqrt{\beta_{z_{i}} \beta_{z_{i+1}}} \sin ^{2}\left(\psi_{z_{i+1}}-\psi_{z_{i}}\right)},
$$

and

$$
\tan \delta_{i+1}=\frac{\left(z_{i} / \sqrt{\beta_{z_{i}}}\right) \sin \psi_{z_{i+1}}-\left(z_{i+1} / \sqrt{\beta_{z_{i+1}}}\right) \sin \psi_{z_{i}}}{\left(z_{i} / \sqrt{\beta_{z_{i}}}\right) \cos \psi_{z_{i+1}}-\left(z_{i+1} / \sqrt{\beta_{z_{i+1}}}\right) \cos \psi_{z_{i}}} .
$$

The third error appears in Eq. (24c): We missed $m$ in the first term of the equation. It should read

$$
C_{2 x}=2 A_{2} m-B_{2}+m^{2} B_{2},
$$

The fourth error appears in Eq. (25d): There is a sign error in the numerator. The equation should read

$$
B_{2}=-\frac{C_{2 x}-2 C_{2 y} m-C_{2 x} m^{2}}{1+2 m^{2}+m^{4}},
$$

We used the correct equations in our codes, therefore the results and conclusions of the article are unchanged. We apologize for the mistakes.

\footnotetext{
*jfcardona@unal.edu.co
} 